\title{
Model Predictive Torque Control with Synchronized Sampling Frequency for High Frequency Induction Machine Drives
}

\author{
Péter Stumpf, István Bara \\ Department of Automation and Applied Informatics \\ Budapest University of Technology and Economics \\ Budapest, Hungary, stumpf@aut.bme.hu
}

\begin{abstract}
Model Predictive Control (MPC) is a promising technique for control of electric drives, as it enables to optimize multiple parameters and offers operation with non-linear systems. Implementation of MPC for high speed/high-pole drives with high rated fundamental frequency - from few hundred up to thousand $\mathrm{Hz}$ - poses many challanges due to the low $G$ sampling to fundamental frequency ratio around the rated speed of the machine. The paper proposes a novel MPC scheme, where the sampling frequency is sychronized to the fundamental frequency to improve the performance. The algorithm can be implemented on a digital device by the recursive equations given in the paper. As it is shown, the method can reduce the number of switchings, achieve better harmonic performance and reduce the torque ripple at the same sampling frequency without synchronization at low $G$ values.
\end{abstract}

Index Terms-Model predictive control, Induction machine, High speed drive

\section{INTRODUCTION}

Nowadays, thanks to dramatic increase of computational power of digital devices, Model Predictive Control (MPC) is a very promising strategy for control of power converters and electrical drives [1], [2]. MPC scheme, contrary to Field Oriented Control (FOC) or Direct Torque Control (DTC), has a simpler concept of design [3]. The control actions are evaluated via a cost function, where system constrains can be included. MPC controlled drives have a fast dynamic performance and good torque response, but generally they operate with variable switching frequency as DTC controlled drives. Papers [3] and [4] offer a detailed comparison between the performance of MPC and FOC or DTC.

The MPC scheme for control of induction motor drive can be classified into two realizations: model predictive torque control (MPTC) and model predictive current control (MPCC). MPTC scheme controls the torque and the stator flux of the machine similar to DTC. MPCC algorithm controls the real and imaginary component of stator current, which is similar to FOC. This paper deals with the MPTC technique.

MPC in electric drives can be used in a wide spectrum of applications. Many modifications and extensions to the

This paper was supported by the János Bolyai Research Scholarship of the Hungarian Academy of Sciences. Research supported by the National Research, Development and Innovation Office (NKFIH) under the grant FK 124913. traditional schemes were published in the recent years depending on the drive conditions or usage. Paper [5] proposed a sensorless MPTC method, where a Model reference adaptive system (MRAS), based on a sliding mode stator voltage model observer, estimates the rotor speed. A new and very simple strategy for MPC based torque and flux control for induction machies is proposed in [6].

The application of MPC for multilevel inverter and direct matrix converter is discussed in [7] and in [8], respectively. Paper [9] proposes an MPC scheme for six-phase induction machine drive with fixed switching frequency by adding a modulation stage. In conventional MPTC only one voltage vector is selected. To obtain better steady-state performance, the concept of duty cycle control was introduced in MPTC by applying nonzero and a zero voltage vector as well. A novel method to optimize the switching instant between the nonzero and zero voltage vector is introduced in [10].

Nowadays increasing attention has been paid to high speed/high-pole drives with high rated fundamental frequency (from few hundred up to thousand $\mathrm{Hz}$ ). The digital control of them poses many challenges due to the limited sampling and switching frequency. Its outcome is that the ratio of the $f_{s}$ sampling frequency and the $f_{1}$ actual fundamental frequency $G=f_{s} / f_{1}$ around the maximum speed of a high speed/highpole motor is a low number. The low value of $G$ can cause stability problems and sampling error in the regulation loop. Furthermore, as the switching frequency in digitally controlled drives is proportional to the sampling frequency, the low $G$ ratio can cause highly distorted current signal as well. This effect can be futher enhanced by the fact that the inductance of high frequency machines is designed to be small compared to ordinary motors.

In the literature MPC algorithms most commonly evaluated for induction machines with standard fundamental frequency of 50 or $60 \mathrm{~Hz}$ and with sampling frequencies between 10 and $50 \mathrm{kHz}$. In this case the value of $G$ is a few hundred and its effect on the performance can be neglected. Some paper, like [11] or [12], deal with the application of MPC for medium and high power drive systems, where the thermal limitation of semiconductor devices restricts the switching frequency resulting in highly distorted current signals. However, the value of $G$ in this cases can be large enough. Paper 
In the current paper some modifications are suggested to the traditional MPTC structure. The most important novelty of the scheme is to apply variable sampling frequency, which is synchronized to the fundamental frequency by keeping the $G=f_{s} / f_{1}$ ratio of sampling frequency and the fundamental frequency a whole number. As the equivalent switching frequency of the MTPC algorithm is proportional to $f_{s}$, by trying to keep $G$ constant the switching frequency $f_{s w}$ is also forced to be synchronized to $f_{1}$. In this way the $m_{f}=f_{s w} / f_{1}$ frequency ratio is also forced to be whole number which is a general rule of thumb in carrier based PWM techniques for low pulse ratios to avoid low order harmonics.

According to [1] selecting the sampling interval as a tuning parameter to set the switching frequency is a poor design choice. At the same time, the proposed synchronization can improve the performance (like lower number of switching, lower harmonic distortion, lower torque ripple) comparing the results at the same sampling frequency without synchronization.

\section{Mathematical BACKGROUND}

MPTC method has three main steps: estimation of the nonmeasurable state variables, prediction for the selected variables and evaluation of the cost function(s) to produce the control signal. For the first step it is essential to have a proper mathematical model of the drive system. As MPC method is inherently implemented in the digital domain the model of the drive in the continuous time domain has to be discretized.

\section{A. Dynamic model of IM}

The operation of a squirrel cage induction machine in a rotating reference frame (RRF), which rotates with an arbitrary selected $\omega_{R}$ angular speed, can be described by the following two differential equations expressing the stator and rotor voltage balance

$$
\begin{aligned}
& \mathbf{v}_{s}=R_{s} \mathbf{i}_{s}+\frac{d \mathbf{\Psi}_{s}}{d t}+j \omega_{R} \boldsymbol{\Psi}_{s} \\
& \mathbf{v}_{r}=\mathbf{0}=R_{r} \mathbf{i}_{r}+\frac{d \mathbf{\Psi}_{r}}{d t}-j\left(\omega_{R}-\omega\right) \boldsymbol{\Psi}_{r}
\end{aligned}
$$

and by the stator $\boldsymbol{\Psi}_{s}$ and rotor $\boldsymbol{\Psi}_{r}$ flux relations

$$
\begin{aligned}
& \boldsymbol{\Psi}_{s}=L_{s} \mathbf{i}_{s}+L_{m} \mathbf{i}_{r} \\
& \boldsymbol{\Psi}_{r}=L_{m} \mathbf{i}_{s}+L_{r} \mathbf{i}_{r},
\end{aligned}
$$

where $\omega$ is the rotor electrical angular velocity. $R_{s}$ and $R_{r}$ are the resistance in one stator and rotor phase, respectively. The total inductance of the stator and rotor can be given as $L_{s}=L_{m}+L_{l s}$ and $L_{r}=L_{m}+L_{l r}$, where $L_{l s}$ and $L_{l r}$ denote the leakage inductance of the stator and rotor. The electric torque of the machine can be given as

$$
M=\frac{3}{2} P \boldsymbol{\Psi}_{s} \times \mathbf{i}_{s},
$$

where $P$ is the number of pole-pairs.

\section{B. Discretization}

By using the forward Euler approximation for the derivatives for a sampling time denoted by $T_{s}$, that is,

$$
\frac{d x}{d t} \approx \frac{x\left(k T_{s}+T_{s}\right)-x\left(k T_{s}\right)}{T_{s}}
$$

the following equation for the prediction of stator flux vector in stationary reference frame (SRF) $\left(\omega_{R}=0\right)$ can be obtained from (1)

$$
\boldsymbol{\Psi}_{s}^{p}\left(k T_{s}+T_{s}\right)=\hat{\mathbf{\Psi}}_{s}\left(k T_{s}\right)+\mathbf{v}_{s}\left(k T_{s}\right) T_{s}-R_{s} \mathbf{i}_{s}\left(k T_{s}\right),
$$

where superscript $p$ denotes "prediction" and ^presents estimation, as flux values due to complexities are not measured directly.

The stator current vector in SRF can be predicted as

$$
\begin{aligned}
\mathbf{i}_{s}^{p}\left(k T_{s}+T_{s}\right)= & \left(1-\frac{R_{e} T_{s}}{\sigma L_{s}}\right) \mathbf{i}_{s}\left(k T_{s}\right)+\frac{T_{s}}{\sigma L_{s}} \mathbf{v}_{s}\left(k T_{s}\right)+ \\
& -j \frac{L_{m}}{\sigma L_{s} L_{r}} T_{s} \omega \hat{\mathbf{\Psi}}_{r}\left(k T_{s}\right)+\frac{L_{m} R_{r}}{\sigma L_{r}^{2} L_{s}} T_{s} \hat{\mathbf{\Psi}}_{r}\left(k T_{s}\right),
\end{aligned}
$$

where $R_{e}=R_{s}+\frac{L_{m}^{2} R_{r}}{L_{r}^{2}}$ and $\sigma=1-\frac{L_{m}^{2}}{L_{r} L_{s}}$. For the prediction of the stator current vector the estimated value of rotor flux vector is necessary, which can be calculated as

$$
\left.\hat{\mathbf{\Psi}}_{r}\left(k T_{s}\right)=\frac{L_{r}}{L_{m}}\left(\hat{\mathbf{\Psi}}_{s}\left(k T_{s}\right)\right)-\sigma L_{s} \mathbf{i}_{s}\left(k T_{s}\right)\right) .
$$

Finally, the electromagnetic torque can be predicted as

$$
\begin{aligned}
M_{e}^{p}(k+1)= & \frac{3}{2} P \boldsymbol{\Psi}_{s}^{p}\left(k T_{s}+T_{s}\right) \times \mathbf{i}_{s}^{p}\left(k T_{s}+T_{s}\right) \\
= & \frac{3}{2} P\left(\Psi_{s \alpha}^{p}\left(k T_{s}+T_{s}\right) i_{s \beta}^{p}\left(k T_{s}+T_{s}\right)-\right. \\
& \left.\Psi_{s \beta}^{p}\left(k T_{s}+T_{s}\right) i_{s \alpha}^{p}\left(k T_{s}+T_{s}\right)\right),
\end{aligned}
$$

where $\alpha$ and $\beta$ denote the real and imaginary components of the vectors in SRF.

For high speed drive, where the $G$ ratio is a low number the applied discretization technique can have a great effect on the preformance. Therefore, the selection of the approximation method plays a crucial role. Paper [11] demonstrates that, discretizing the equations by an improved Taylor method has better performances than the one discretized by the Euler method. The method of discretization of the equation of induction machine by using Tustin method is introduced in [13].

\section{Model Predictive Torque Control}

The block diagram of the proposed MPTC method considering in the paper is shown in Fig.1, where an external PI speed controller generates the $M^{*}$ reference value of the electric torque. The proposed modifications are denoted by red letters. 


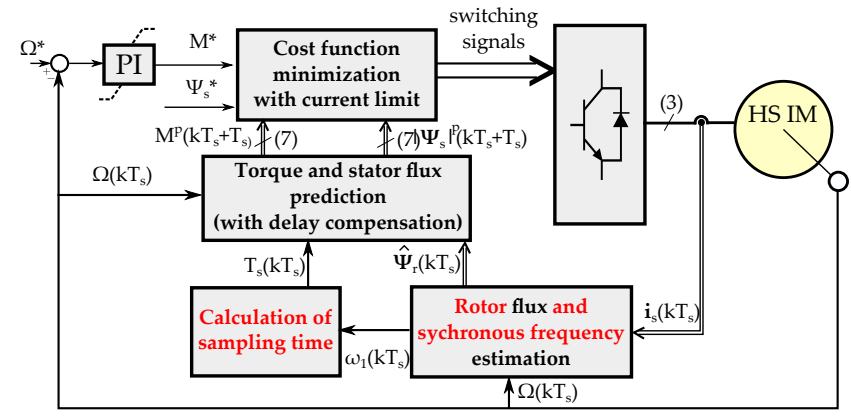

Fig. 1. Control diagram of proposed MTPC

\section{A. Scheme with weighting factor}

The traditional scheme predicts both the electromagnetic torque and the stator flux for all possible voltage vectors by using (7) and (10). In the case of two-level inverter the number of different possible voltage vectors is 7 . One method to select most appropiate voltage vector is to minimize of a cost function, which can be expressed as a linear combination of torque and stator flux errors:

$$
g=\left|M^{*}-M^{p}\left(k T_{s}+T_{s}\right)\right|+k_{\Psi}\left|\Psi_{s}^{*}-\right| \Psi_{s}^{p}\left|\left(k T_{s}+T_{s}\right)\right|
$$

where $k_{\Psi}$ is the so-called weighting factor.

The value of the weighting factor has great impact on the performance of the drive and its tuning is a nontrivial process, which is generally based on heuristic procedure. Many paper in the literature deal with the proper tuning of $k_{\Psi}$. Paper [14] introduced a technique to optimize the value of $k$ in real time to reduce torque ripple.

\section{B. Scheme without weighting factor}

Another way, which is investigated in the current paper, is to evaluate the torque and flux error separately by using two cost functions [15]

$$
\begin{aligned}
& g_{1}=\left|M^{*}-M^{p}\left(k T_{s}+T_{s}\right)\right|^{2} \\
& g_{2}=\left|\Psi_{s}^{*}-\right| \Psi_{s}^{p}\left|\left(k T_{s}+T_{s}\right)\right|^{2}
\end{aligned}
$$

The objective functions $g_{1}$ and $g_{2}$ are evaluated for each possible voltage vector. Then the voltage vectors are sorted and ranked based on the value of error: voltage vectors with lower error are assigned a lower ranking. $r_{1}$ and $r_{2}$ denotes the ranking value related to $g_{1}$ and $g_{2}$, respectively. The ranking value expresses a relative quality of the voltage vector compared to the other possible voltage vectors. Finally, the voltage vector with the minimum average value of its rankings is selected as

$$
\mathbf{v}_{\text {opt }}\left(k T_{s}\right)=\arg \min _{\mathbf{v}_{1} \ldots \mathbf{v}_{7}} \frac{r_{1}+r_{2}}{2} .
$$

It results in an equal compromise of tracking for torque and flux at the same time.

By using the presented ranking approach, it is possible for multiple voltage vectors to have the same averaged ranking [15]. To solve this issue, priorities can be assigned for each objective but only for the condition of multiple optimal voltage vectors. In the paper, for multiple otimal voltage vectors the vector which minimizes the torque error is selected.

\section{Consideration of calculation time}

One well-known disadvantage of real-time digital implementation of MPC techniques is the required high processing capability due to the large number of calculations. It results in a delay between the measurements and the actuation, which can deteoriate the performance of the drive if it is not considered and not compensated. The principle for delay compensation for MPC can be found in [16].

The behavior of a real-time implementation is visualized in Fig.2(a). At the start of the $k^{t h}$ sampling period the stator current and the speed of the machine is measured. The calculation of MPTC is finished at $k T_{s}+t_{\text {calc }}$. Generally $t_{\text {calc }}$ is not a fixed time. Therefore, most commonly, the calculated optimal voltage vector $\mathbf{v}_{\text {opt }}\left(k T_{s}\right)$ is applied at the start of the next sampling period at $k T_{s}+T s$ time instant. Consequently, at $k T_{s}$ the previously calculated voltage vector $\mathbf{v}_{\text {opt }}\left(k T_{s}-T_{s}\right)$ is applied. It results in a one sampling time delay in the control loop.

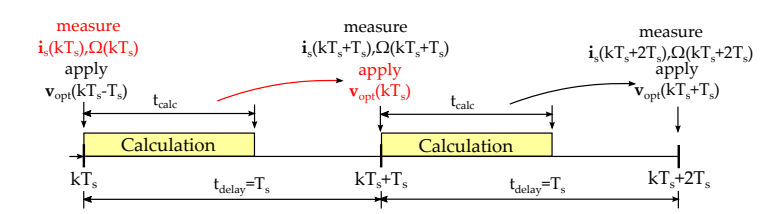

(a) $t_{d}=T_{s}$

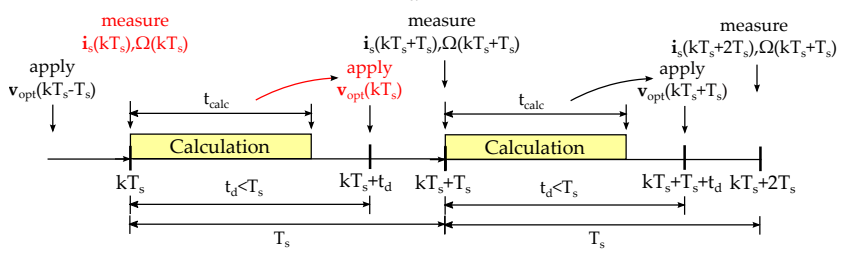

(b) $t_{d} \leq T_{s}$

Fig. 2. Time sequence of discrete implementation of MPTC

The model-based compensation method predicts the value of the control variables (like stator and rotor flux and stator current) at $k T_{s}+T_{s}$ time instant using the measured values in the $k^{\text {th }}$ sampling period and $\mathbf{v}_{\text {opt }}\left(k T_{s}-T_{s}\right)$. It means that the system equations has to be calculated once more before the actual optimization can be started. Practically, the optimization is carried out in $k^{t h}$ period for $\left(k T_{s}+2 T_{s}\right)$ time instant [10], [16].

As it can be seen on Fig.2(b), it is possible, if $t_{\text {calc }}$ smaller than $T_{s}$, to apply the calculated voltage vector $\mathbf{v}_{\mathbf{~ o p t}}(k)$ earlier than the next sampling period, at time instant $k T_{s}+t_{d}$, where $t_{d}$ is a fixed constant value. Here it should be ensured that $t_{\text {calc }}<t_{d} \leq T_{s}$. In this case the compensation should be carried out by using $t_{d}$ in the equations.

In the current paper, as it will be shown later, $T_{s}$ sampling time is not constant and varies with the electrical frequency. Therefore, the time sequence presented on Fig.2(b) is assumed and $t_{d}$ will be a parameter. 
Equations for the compensation of $t_{d}$ will be given in the following section.

\section{Proposed SynChronized MPTC SCHEME}

In the current paper some modifications are suggested to the traditional MPTC structure. As it was mentioned previously the most important novelty of the scheme is to apply variable sampling time, which is synchronized to the fundamental frequency. Thus the $G$ ratio of sampling frequency and the fundamental frequency is a whole number. It acts as a parameter for the algorithm.

\section{A. Calculation of fundamental frequency}

In traditional MPTC structure the stator flux is calculated using only (7). In the paper it is suggested to use the so-called current model, where the rotor flux is calculated based on the stator current.

By selecting the angular speed of RRF to be the mechanical angular speed $\omega_{R}=\omega$, (1)-(4) can be simplified to

$$
\begin{aligned}
\frac{d \boldsymbol{\Psi}_{r}}{d t} & =\frac{R_{r} L_{m}}{L_{r}} \mathbf{i}_{s}-\frac{R_{r}}{L_{r}} \boldsymbol{\Psi}_{r} \\
\boldsymbol{\Psi}_{s} & =\sigma L_{s} \mathbf{i}_{s}+\frac{L_{m}}{L_{r}} \boldsymbol{\Psi}_{r},
\end{aligned}
$$

This method has the advantage over the previous one, that it applies a closed-loop integrator. It requires the mechanical angle for coordinate transformation, which can be obtained by integration.

As the value of the rotor flux is crucial to have an accurate and stable response the so-called trapezoidal (Tustin) integral approximation is suggested [13]. The discrete integral can be calculated as follows between two consecutive time steps

$$
f\left(k T_{s}+T_{s}\right)-f\left(k T_{s}\right)=\int_{k T_{s}}^{k T_{s}+T_{s}} g(\tau) d \tau,
$$

It should be noted, this observer can estimate the present value of the rotor flux in the $k^{\text {th }}$ period. Therefore (17) should be calculated between $(k-1) T s$ and $k T_{s}$ instants.

Applying (17) to (15)

$$
\begin{gathered}
\Psi_{r}\left(k T_{s}\right)-\Psi_{r}\left(k T_{s}-T_{s}\right)=\frac{R_{r} L_{m}}{L_{r}} \int_{k T_{s}-T_{s}}^{k T_{s}} \mathbf{i}_{s}(\tau) d \tau- \\
\frac{R_{r}}{L_{r}} \int_{k T_{s}-T_{s}}^{k T_{s}} \boldsymbol{\Psi}_{r}(\tau) d \tau
\end{gathered}
$$

The sinusoidal stator current viewed in the RRF appears to be a slow moving sinusoidal signal at the slip frequency and can be modelled as a ramp signal with an average value of $\left(\mathbf{i}_{s}\left(k T_{s}\right)+\mathbf{i}_{s}\left(k T_{s}-T_{s}\right)\right) / 2$.

The flux integral is approximated with the trapezoid according to Tustin definition as

$$
\int_{k T_{s}-T_{s}}^{k T_{s}} \boldsymbol{\Psi}_{r}(\tau) d \tau=\frac{T_{s}}{2}\left(\boldsymbol{\Psi}_{r}\left(k T_{s}\right)+\boldsymbol{\Psi}_{r}\left(k T_{s}-T_{s}\right)\right) .
$$

The discrete version of the rotor flux estimator

$$
\hat{\mathbf{\Psi}}_{r}\left(k T_{s}\right)=K_{1} \hat{\mathbf{\Psi}}_{r}\left(\left(k T_{s}-T_{s}\right)\right)+K_{2}\left(\mathbf{i}_{s}\left(k T_{s}\right)+\mathbf{i}_{s}\left(k T_{s}-T_{s}\right)\right)
$$

where

$$
K_{1}=\frac{1-\frac{R_{r} T_{s}}{2 L_{r}}}{1+\frac{R_{r} T_{s}}{2 L_{r}}} \quad \text { and } \quad K_{2}=\frac{\frac{R_{r} L_{m} T_{s}}{2 L_{r}}}{1+\frac{R_{r} T_{s}}{2 L_{r}}}
$$

After estimating $\hat{\mathbf{\Psi}}_{r}\left(k T_{s}\right)$ in the RRF, it is transformed back to SRF. The actual $\rho$ angle of the rotor flux vector, which provides the angle of the field, in SRF can be calculated by using $\operatorname{atan} 2()$ function. By using $\rho$ the stator current vector can be transformed to RRF, in literature denoted by $d-q$ coordinate system, similar as in indirect FOC scheme.

The fundamental frequency in the $k^{t h}$ period can be estimated as

$$
f_{1}\left(k T_{s}\right)=\frac{1}{2 \pi}\left(\omega\left(k T_{s}\right)+\frac{R_{r} L_{m}}{L_{r}} \frac{1}{\left|\hat{\Psi}_{r}\left(k T_{s}\right)\right|} i_{s q}\left(k T_{s}\right),\right.
$$

where $i_{s q}\left(k T_{s}\right)$ is imaginary part of the stator current vector in the $d-q \mathrm{RRF}$ also called as torque producing component.

As the sampled current contains higher harmonics originating from the switching, the estimated $f_{1}$ fundamental frequency should be filtered with simple low-pass filter. Generally, low-pass filtering of signals in closed-loop application reduces the performance or even can cause instability. However, in this case the value of $f_{1}$ is not directly used by the control variables, so the filtering does not degrade the performance of the drive.

The sampling frequency for the $k^{t h}$ period can be obtained by

$$
T_{s}\left(k T_{s}\right)=\frac{1}{f_{1 f}\left(k T_{s}\right)} G,
$$

where $f_{1 f}$ is the filtered value of the fundamnetal frequency and $G$ is the ratio of sampling frequency and the fundamental frequency. The value of $G$ can be constant or it can be changed as the function of $f_{1}$ to limit the sampling frequency.

\section{B. Stator flux prediction}

In traditional MPTC scheme the stator flux is predicted/calculated by using (7) and the rotor flux is obtained from the stator flux as (9). In the proposed sychronized MTPC the rotor flux should be estimated to calculate the fundamental freqeuncy (see (20)). Therefore, its value can be utilized during the calculations.

For the stator flux calculation the following steps should be carried out. First, the effect of computational time should be compensated. By assuming that, the amplitude of the rotor flux is constant during $t_{d}$ (the time constant of the rotor flux is considerably higher than $t_{d}$ ), the value of $\hat{\mathbf{\Psi}}_{r}$ can be calculated for the $k T_{s}+t_{d}$ time instant as

$$
\hat{\mathbf{\Psi}}_{r}\left(k T_{s}+t_{d}\right)=\hat{\mathbf{\Psi}}_{r}(k) e^{j \omega_{1} T_{d}} \approx \hat{\mathbf{\Psi}}_{r}(k) e^{j \omega T_{d}}
$$

as for high speed drives the slip speed is small compared to the fundamental angular frequency and $\omega_{1} \approx \omega$ (and assuming that the number of pole pairs is 1 ). 
From (24) the stator flux $\hat{\mathbf{\Psi}}_{s}\left(k T_{s}+t_{d}\right)$ at $k T_{s}+t_{d}$ time instant can be calculated by rewritting (7) as

$$
\hat{\mathbf{\Psi}}_{s}\left(k T_{s}+t_{d}\right)=\frac{L_{m}}{L_{r}} \hat{\mathbf{\Psi}}_{r}\left(k T_{s}+t_{d}\right)+\sigma L_{s} \hat{\mathbf{i}}_{s}\left(k T_{s}+t_{d}\right)
$$

The value of $\hat{\mathbf{\Psi}}_{s}\left(k T_{s}+t_{d}\right)$ can be used to predict the stator flux for all the possible voltage vectors based on (7) for the $k T_{s}+T_{s}+t_{d}$ time instant.

The term $\hat{\mathbf{i}}_{s}\left(k T_{s}+t_{d}\right)$ in (25) is the estimated stator current vector at $k T_{s}+t_{d}$ time instant to compensate the effect of calculation time. It can be obtained as

$$
\begin{aligned}
\hat{\mathbf{i}}_{s}\left(k T_{s}+t_{d}\right)= & \left(1-\frac{R_{e} t_{d}}{\sigma L_{s}}\right) \mathbf{i}_{s}(k)+\frac{t_{d}}{\sigma L_{s}} \mathbf{v}_{o p t}(k-1)+ \\
& -j \frac{L_{m}}{\sigma L_{s} L_{r}} t_{d} \omega \hat{\mathbf{\Psi}}_{r}(k)+\frac{L_{m} R_{r}}{\sigma L_{r}^{2} L_{s}} t_{d} \hat{\mathbf{\Psi}}_{r}(k) .
\end{aligned}
$$

The value of $\hat{\mathbf{i}}_{s}\left(k T_{s}+t_{d}\right)$ can be used to predict the stator current for all the possible voltage vectors based on (8) for the $k T_{s}+T_{s}+t_{d}$ time instant.

As in the proposed algorithm $T_{s}$ is not constant, its actual $T_{s}=T_{s}\left(k T_{s}\right)$ value (see (23)) should be used in the equations. Naturally, the previously presented equations for delay compensation can be used for $t_{d}=T_{s}$ as well.

\section{Flow diagram of the proposed technique}

Figure 3 presents the flow diagram of the proposed syncrhonized MPTC technique by giving the exact equations as well.

\section{Simulation Results}

Simulation analysis in Matlab/Simulink environment were carried out an induction machine with a rated speed of 18 $\mathrm{krpm}$ to verify the operation of the algorithm. The rated data and main parameters of the simulated high frequency machine were: power: $P_{n}=3 \mathrm{~kW}$, rms line-to-line voltage $V_{L L, R M S}=$ $380 \mathrm{~V}$, phase current $I_{n}=7.7 \mathrm{~A}$, rated frequency $f_{1 n}=300$ $\mathrm{Hz}$, stator and rotor resistance $R_{s}=1.125 \Omega, R_{r}=0.85 \Omega$, stator and rotor leakage inductance $L_{l s}=2.5 \mathrm{mH}$ and $L_{l r}=$ $1.4 \mathrm{mH}$, magnetizing inductance $X_{m}=45 \mathrm{mH}$ and the number of pole pairs is $P=1$. The DC link voltage of the inverter was $V_{D C}=540 \mathrm{~V}$. During the simulation the delays occuring in a microcontroller or DSP based system were also taken into consideration. During the simulation $t_{d}$ was selected to be $40 \mu s$.

\section{A. Steady state}

First the performance of the proposed synchronized MPTC technique is demonstrated in steady-state. The reference speed is selected to be $n^{*}=15000 \mathrm{rpm}\left(\Omega^{*}=1570.8 \mathrm{rad} / \mathrm{sec}\right)$ and the machine is loaded with its rated torque $\left(M_{\text {load }}=1.5\right.$ $\mathrm{Nm}$ ). Figure 4(a) presents the time function of the stator phase current $i_{s a}$ and its Fourier spectra, when $G=59$ $\left(f_{1} \approx 255 \mathrm{~Hz}\right)$. For the better comparison the same diagrams are plotted for unsynchronized case on Fig.4(b), where the

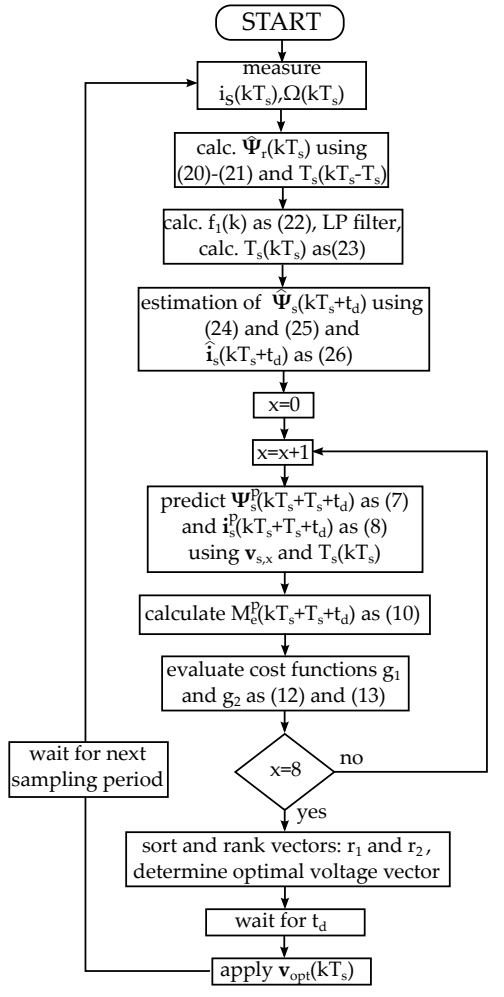

Fig. 3. Flow diagram of the proposed MPTC technique

sampling frequency is set to $15 \mathrm{kHz}$, which is practically the same as for sychronized case. As it can be seen thanks for the synchronization the stator current repeats itself period by period, while a low order harmonics can be seen for unsynchronized case on Fig.4(b). The existence of the low order harmonics for unsynchronized case is verified by the Fourier spectra as well.

The switching frequency is estimated by calculating the number of switching in steady-state for $1 \mathrm{sec}$. For synchronized case the calculated equivalent switching frequency is $7315 \mathrm{~Hz}$, while for unsychronized case it is $7868 \mathrm{~Hz}$. It results that, a better harmonic performance can be obtained with a lower number of switching using the propsed synchronized MPTC scheme.

Figure 5(a) presents the time function of the stator phase current $i_{s a}$ and the electric torque, when the sampling to fundamental frequency is only $G=30$. For the comparison the same diagrams are plotted again for unsynchronized case on Fig.5(b), where the sampling frequency is set to $7.7 \mathrm{kHz}$, which is practically the same as for sychronized case. Due to the low value of $G$ the distortion in the current signals is much larger than in the previous case resulting in large torque ripples. For the proposed synchronized case the current signal again repeats itself period by period resulting in a much smaller torque ripple. For the unsyhcronized case a low order harmonic, with much larger amplitude than the previous case, can be seen clearly on the time diagram. It results in a considerably higher ripple in the torque signal. 


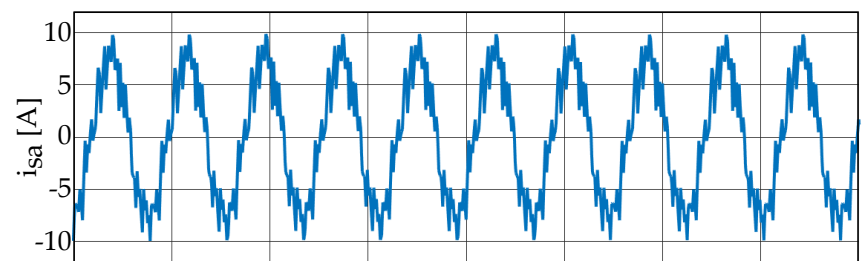

time: $5 \mathrm{~ms} / \mathrm{div}$

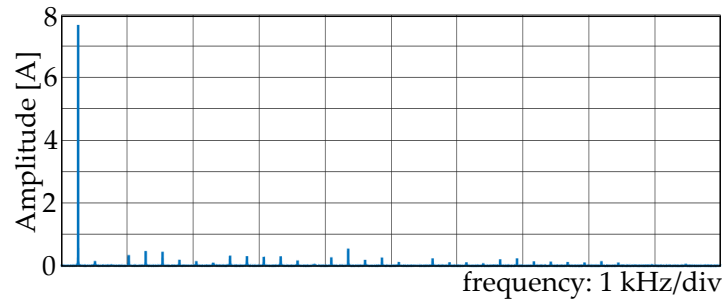

(a) Proposed synchronized MPTC, $G=59, f_{s} \approx 59 \cdot 255 H z \approx 15 \mathrm{kHz}$
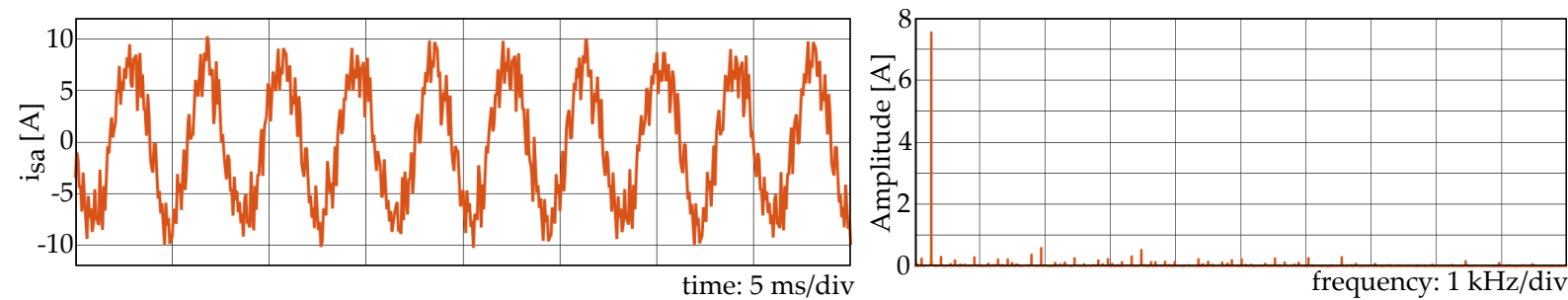

(b) MPTC without sychronization, $f_{s}=15 \mathrm{kHz}$

Fig. 4. Stator phase current and its Fourier spectra, simulation results, $n^{*}=15000 \mathrm{rpm}, M_{\text {load }}=1.5 \mathrm{Nm}$

The difference between the calculated equivalent switching frequencies for synchronized and unsychronized case is much smaller for $G=30$, it is around $4 \mathrm{kHz}$ for both cases. It results that the switching to fundamental frequency ratio is around 15 .

\section{B. Transient performance}

Figure 6 presents the simulation results during start-up and for sudden torque change at $t=1.05 \mathrm{sec}$. The reference speed again is set to $n^{*}=15000 \mathrm{rpm}$. The MPTC scheme starts with fixed sampling frequency of $f_{s}=10 \mathrm{kHz}$, and after reaching the fundamental frequency of $f_{1}=90 \mathrm{~Hz}$ the synchronization is turned on with a ratio of $G=100$. As the fundamental frequency increases the $G$ is decreased step by step at certain fundamental frequencies to obtain an average sampling frequency between 10 and $12 \mathrm{kHz}$. The time function of the stator current is zoomed for each $G$ value. As it can be seen the torque ripple is smaller for the synchronized case. The current signal are not exactly synchronized in transient when $f_{1}$ is not constant. However, it still has a good harmonic performance and when the steady-state is reached the current becomes synchronized similar to Fig.4(a) and Fig.5(a).

\section{CONCLusions}

The paper focuses on the implementation issues of MTPC algorithm on high speed/high-pole drives with high rated fundamental frequency from few hundred up to thousand Hz. In this case the ratio of the $f_{s}$ sampling frequency and the $f_{1}$ actual fundamental frequency $G=f_{s} / f_{1}$ around the maximum speed motor is a low number.

The paper introduces the theoretical background of traditional MPTC schemes. Additionally, a novel MPC scheme, where the sampling frequency is sychronized to the fundamental frequency, is proposed. Furthermore, the stator flux is predicted using the estimated rotor flux, which is calculated from the current model using Tustin approximation. The algorithm can be implemented on a digital device by the recursive equations and the flow chart presented in the paper.

The performance of the method is demonstrated by simulation results in steady-state and in transient. Based on the results it can be concluded, the proposed synchronized MPTC scheme can eliminate the low order harmonics and the number of switching can be reduced comparing to unsynchronized case at the same sampling frequency. Furthermore, the ripple in the electric torque can be smaller. As the main conclusion, at low $G$ values it is worth to synchronize the sampling frequency to the fundamental one to obtain better performance.

The laboratory implementation and the experimental results will be discussed in another paper.

\section{REFERENCES}

[1] P. Karamanakos and T. Geyer, "Guidelines for the design of finite control set model predictive controllers," IEEE Transactions on Power Electronics, vol. 35, no. 7, pp. 7434-7450, 2020.

[2] S. Vazquez, J. Rodriguez, M. Rivera, L. G. Franquelo, and M. Norambuena, "Model predictive control for power converters and drives: Advances and trends," IEEE Transactions on Industrial Electronics, vol. 64, no. 2, pp. 935-947, Feb 2017.

[3] F. Wang, Z. Zhang, X. Mei, J. Rodriguez, and R. Kennel, "Advanced control strategies of induction machine: Field oriented control, direct torque control and model predictive control," Energies, vol. 11, no. 1, 2018.

[4] P. Karlovsky and J. Lettl, "Indution motor drive direct torque control and predictive torque control comparison based on switching pattern analysis," Energies, vol. 11, no. 7, 2018.

[5] F. Wang, S. A. Davari, Z. Chen, Z. Zhang, D. A. Khaburi, J. Rodrguez, and R. Kennel, "Finite control set model predictive torque control of induction machine with a robust adaptive observer," IEEE Transactions on Industrial Electronics, vol. 64, no. 4, pp. 2631-2641, April 2017.

[6] M. Norambuena, J. Rodriguez, Z. Zhang, F. Wang, C. Garcia, and R. Kennel, "A very simple strategy for high-quality performance of ac machines using model predictive control," IEEE Transactions on Power Electronics, vol. 34, no. 1, pp. 794-800, 2019.

[7] N. Oikonomou, C. Gutscher, P. Karamanakos, F. D. Kieferndorf, and T. Geyer, "Model predictive pulse pattern control for the five-level active neutral-point-clamped inverter," IEEE Transactions on Industry Applications, vol. 49, no. 6, pp. 2583-2592, Nov 2013. 


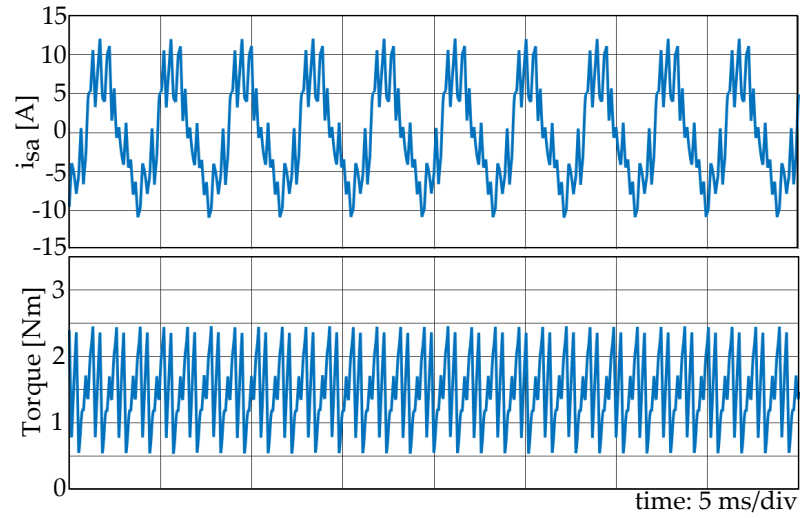

(a) Proposed synchronized MPTC, $G=30, f_{s} \approx 30 \cdot 255 \approx 7,6$ $\mathrm{kHz}$

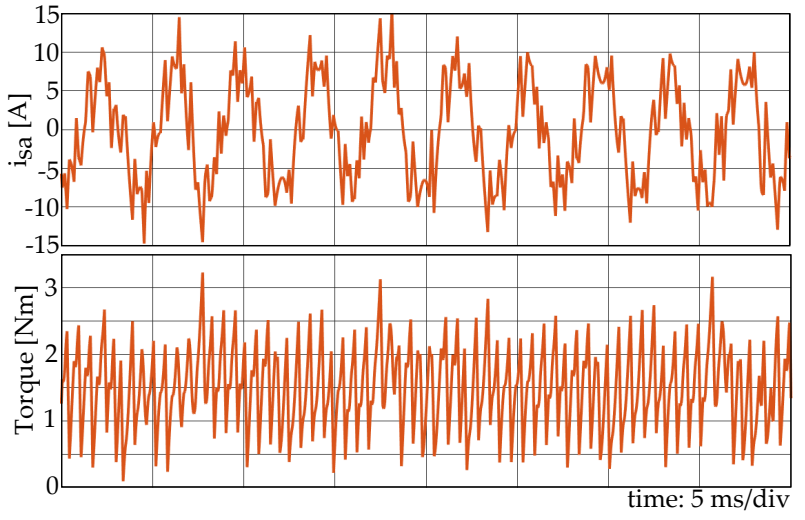

(b) MPTC without sychronization $f_{s}=7.7 \mathrm{kHz}$

Fig. 5. Stator phase current and electric torque, simulation results, $n^{*}=15000 \mathrm{rpm}, M_{l o a d}=1.5 \mathrm{Nm}$

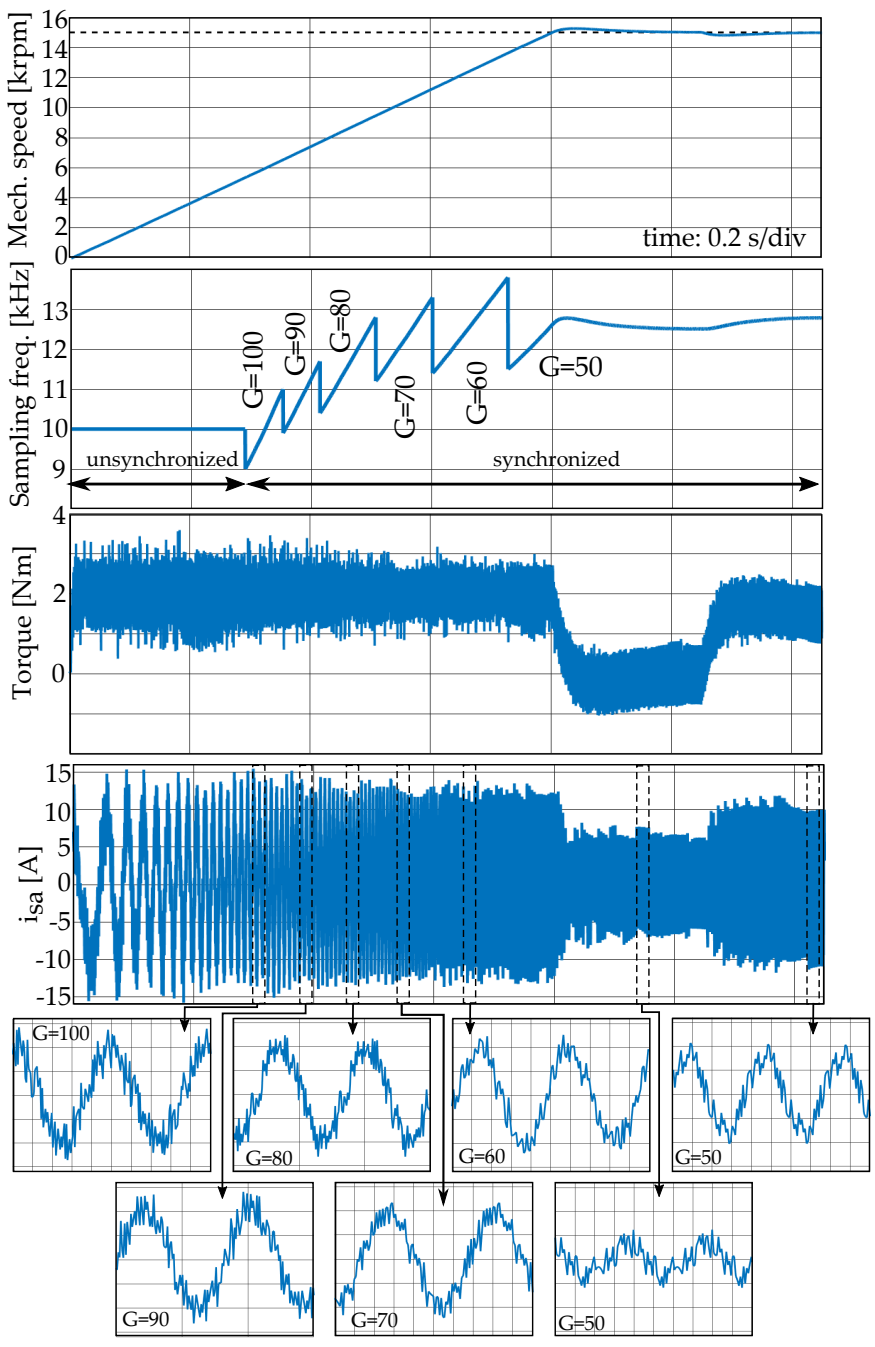

Fig. 6. Start-up, simulation results, $n^{*}=15000 \mathrm{rpm}, M_{\text {load }}=1.5 \mathrm{Nm}$

[8] J. Zhang, M. Norambuena, L. Li, D. Dorrell, and J. Rodriguez, "Sequential model predictive control of three-phase direct matrix converter," Energies, vol. 12, no. 2, 2019.
[9] O. Gonzalez, M. Ayala, J. Doval-Gandiy, J. Rodas, R. Gregor, and M. Rivera, "Predictive-fixed switching frequency current control strategy applied to six-phase induction machine," Energies, vol. 12, no. 12, 2019.

[10] Y. Zhang and H. Yang, "Model-predictive flux control of induction motor drives with switching instant optimization," IEEE Transactions on Energy Conversion, vol. 30, no. 3, pp. 1113-1122, Sep. 2015.

[11] Q. Meng, J. Li, H. Li, and Y. Yan, "Model predictive control of induction motors based on improved discretizing method under low switching frequency," in IECON 2017 - 43rd Annual Conference of the IEEE Industrial Electronics Society, Oct 2017, pp. 5144-5149.

[12] P. Acuna, C. A. Rojas, R. Baidya, R. P. Aguilera, and J. E. Fletcher, "On the impact of transients on multistep model predictive control for medium-voltage drives," IEEE Transactions on Power Electronics, vol. 34, no. 9, pp. 8342-8355, Sep. 2019.

[13] P. Stumpf and A. L. Varadi, "Investigation of estimator algorithms for high speed drive systems," in 2018 18th International Conference on Mechatronics - Mechatronika (ME), Dec 2018, pp. 1-8.

[14] S. A. Davari, D. A. Khaburi, and R. Kennel, "An improved fcsmpc algorithm for an induction motor with an imposed optimized weighting factor," IEEE Transactions on Power Electronics, vol. 27, no. 3, pp. 1540-1551, March 2012.

[15] C. A. Rojas, J. Rodriguez, F. Villarroel, J. R. Espinoza, C. A. Silva, and M. Trincado, "Predictive torque and flux control without weighting factors," IEEE Transactions on Industrial Electronics, vol. 60, no. 2, pp. 681-690, Feb 2013.

[16] P. Cortes, J. Rodriguez, C. Silva, and A. Flores, "Delay compensation in model predictive current control of a three-phase inverter," IEEE Transactions on Industrial Electronics, vol. 59, no. 2, pp. 1323-1325, Feb 2012. 